\title{
ARTICLE
}

\section{Radiation shielding and safety systems of the SPring-8 angstrom compact free-electron laser, SACLA}

\author{
Yoshihiro Asano ${ }^{\mathrm{a}^{*}}$ and Toshiro Itoga ${ }^{\mathrm{b}}$ \\ ${ }^{a}$ Safety Design Group SPring-8 Center RIKEN, 1-1,Koto,Sayo, Hyogo-ken,679-5148, Japan; ${ }^{b}$ XFEL Japan Synchrotron Radiation \\ Research Center 1-1,Koto, Sayo, Hyogo-ken, 679-5143, Japan
}

\begin{abstract}
The shielding design of the 8-GeV class X-ray Free-Electron Laser facility at SPring-8, SACLA, has been accomplished by using a semi-empirical code, SHIELD11, and Monte Carlo codes, EGS4 and FLUKA for the bulk shield. These results were compared with each other. The Beamline hutches of SACLA have also been designed and the leakage doses have been estimated including the incident conditions of the abnormal electron aberrance. SACLA is now being operated stably with sub-angstrom oscillations without any radiation problems.
\end{abstract}

Keywords: X-ray free-electron laser; SPring-8; SACLA; Monte Carlo; shielding design; FLUKA; EGS4; SHIELD11; radiation safety

\section{Introduction}

The SPring-8 angstrom compact free-electron laser, SACLA, is the newest X-ray free-electron laser facility, and can now produce the shortest wavelengths of any $\mathrm{X}$-ray laser in the world. SACLA is based on three new technologies: one is the low-emittance thermionic $\mathrm{CeB}_{6}$ electron gun; another is c-band accelerators with gradient of over $35 \mathrm{MV} / \mathrm{m}$; and the third is in-vacuum type undulators with a short period length of $18 \mathrm{~mm}$. The Linac can produce electrons of up to $8.5 \mathrm{GeV}$ energy with repetition rates of $60 \mathrm{~Hz}$ [1].

The SACLA project was started in December 2005, and commissioning started in February 2011. In June 2011, the X-ray laser successfully produced its first radiation with wavelengths of less than $0.1 \mathrm{~nm}$.

\section{SACLA}

Figure 1 shows a schematic diagram of SACLA. Electrons are produced by a $\mathrm{CeB}_{6}$ electron gun with 500 $\mathrm{keV}$ and $1 \mathrm{~A}$, and pulsed 1 nanosecond by a deflector. High peak currents from the initial value of $1 \mathrm{~A}$ to $3 \mathrm{kA}$ are produced by a three-stage compression system (BC1 BC3); these compressors can also remove dark currents. In addition to bunch compressors, one chicane was installed to remove dark currents. SACLA is designed for use in 5 beamlines by utilizing a switching magnet, and now two beamlines (BL-1 for broad-band beams and BL-3 for XFEL) are under operation. The accelerated electrons pass through undulators of about

*Corresponding author. Email: asano@spring8.or.jp
$100 \mathrm{~m}$ length, then go down into a beam dump with an inclination angle of 20 degrees. The laser light beams go straight into optics hutches and experimental hutches through a shielding wall in the direction of the SPring-8 storage ring. The length of the machine, including the experimental hall, is about $700 \mathrm{~m}$. In addition to use in the experimental areas, the $\mathrm{X}$-ray laser beams go through to the XFEL-SR inter-available building for experiments to concurrent use.

\section{Shielding design and calculations}

Based on the ALARA principle and the design criteria of the SPring-8 site, the shielding design of SACLA was achieved by using empirical formula, such as SHIELD 11 [2], and the EGS4 [3] and FLUKA [4] Monte Carlo codes.

\subsection{Accelerator and undulator section}

Using beam-dynamics simulations and data of the $250 \mathrm{MeV}$ SCSS Compact SASE test facility [5], the beam loss distribution is assumed to be $50 \%$ from the electron gun to $50 \mathrm{MeV}$ ( BC1:bunch compressor 1), 10\% from $\mathrm{BC} 1$ up to $440 \mathrm{MeV}$ (BC2), and $1 \%$ at any points from $\mathrm{BC} 2$ to the end of the accelerator section; $0.1 \%$ beam loss is employed for the undulator section, except for the beam dump, because of high-quality electron beam is required to oscillate the X-ray laser. Unwanted beam loss must be avoided in order to protect against radiation damage of the undulator magnets [6]. Based on these assumptions and the design criteria, we chose the thicknesses of the shield tunnel to be $2 \mathrm{~m}$ 


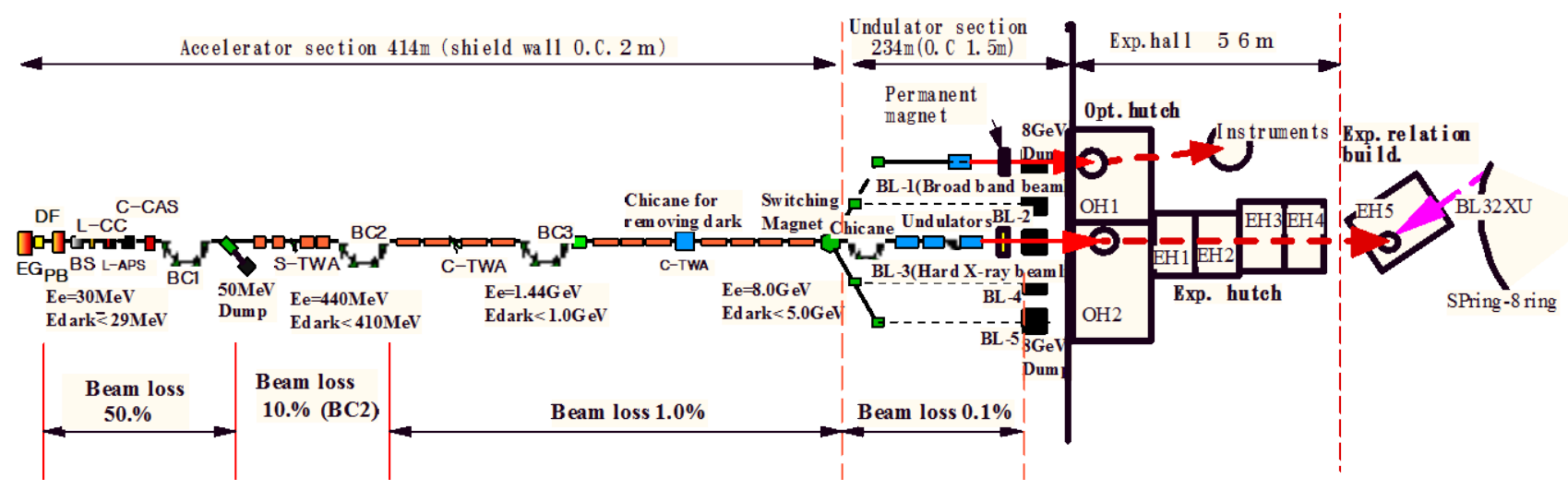

Figure 1. Schematic draw out of SACLA. EG, 500keV electron gun; DF, deflector; BS, booster; L-CC, L-band correction cavity; C-CAS, C-band correction acceleration tube; BC1 BC3, bunch compressor; OH1 \&2, Optics hutch 1 \&2; EH1 4, Experimental hutch 1 4; EH5, XFEL-SR inter-available hutch.

ordinary concrete for the accelerator section, and $1.5 \mathrm{~m}$ for the undulator section. Additional iron plates of 65 $\mathrm{cm}$ at maximum are placed at the top of the dump, as shown in Figure 2 including the dose distribution during electron injection into the dump. The dump has a double cylindrical structure, and the core (inner cylinder) is made of graphite; the outer is made of 40 $\mathrm{cm}$ thick iron. As shown in the figure, it is clear that the leakage dose outside the beamline hole to the optics hutch is negligibly small during $8.5 \mathrm{GeV}$ electron injections to the dump with $30 \mathrm{nC} / \mathrm{s}$. The leakage doses at the roof are lower than the $0.5 \mu \mathrm{Sv} / \mathrm{h}$ during injections, and much lower than the dose limit of the radiation controlled area. Figure $\mathbf{3}$ shows the leakage dose distributions outside roof due to an electron beam loss of $0.1 \%$, in compared with the leakage dose due to injection to the dump by using SHIELD 11. In the figure, the horizontal axis shows the scattering angle from the electron beam transport axis to the estimation points outside of the roof. The beam axis of the electron injections to the dump inclines by 20 degrees, so that the maximum leakage dose including the position due to the injections to the dump shows good agreement with

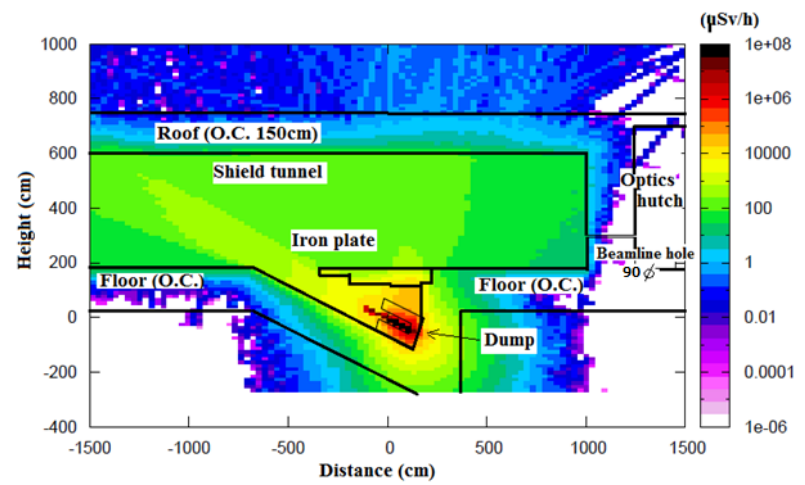

Figure 2. Dose distributions around the SACLA dump area during the injection of $30 \mathrm{nC} / \mathrm{s}$ with $8.5 \mathrm{GeV}$ electrons to the dump. The origin of the graph is set at the beam injection point of the dump; the height of the electron beam is 298.46 $\mathrm{cm}$. O.C. means ordinary concrete. that of the FLUKA calculation, and the neutron dose outside the roof is dominant. The leakage dose outside the roof due to $0.1 \%$ electron beam loss is higher than the dose due to full-beam injection to the dump in this shielding configuration. Due to the design criteria of the dose at the site boundary, the maximum leakage dose outside the roof is also much lower than the dose limit of the radiation-controlled area, and the neutron doses are dominant in both cases, as shown in Figure 3.

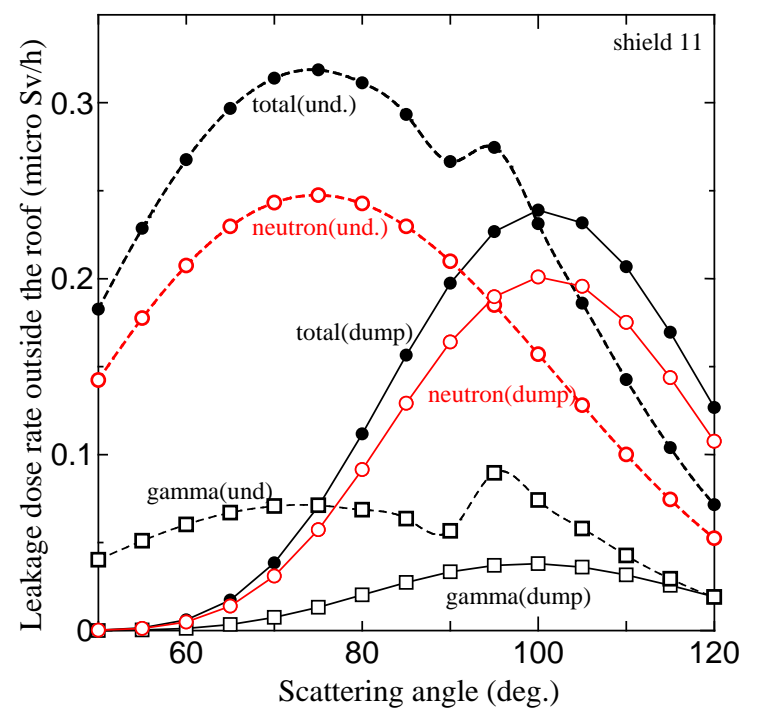

Figure 3. Leakage dose distributions at the roof of the shield tunnel due to injection of the full beam to the dump and $0.1 \%$ of the electrons beam loss. The horizontal axis shows the scattering angles from each electron beam axis. The solid and dotted lines show the dose due to full beam injection to the dump and $0.1 \%$ beam loss, respectively (with full circles, total dose; open circles, neutron dose; open squares, gamma dose).

\subsection{Beamline section}

For the beamline shielding, three radiation sources were considered, as follows: (1) X-ray laser and synchrotron radiation, (2) gas bremsstrahlung and associated photo-neutrons, (3) high-energy radiation due to electron beam loss, including muons. The main 
parameters of SACLA for radiation shielding and safety are listed in Table1, including the SPring-8 parameters. As discussed concerning the characteristics of the XFEL and synchrotron facilities in a previous paper [7], high-energy electrons are always injected into the beam dump, and the beamlines must be constructed in the direction of the movements of electrons at the XFEL. However, the currents of SACLA are $3 \times 10^{-7}$ times lower than that of SPring-8. Therefore, the total photons of the $\mathrm{X}$-ray laser and the synchrotron radiation are lower than that of SPring-8, even with the extremely high photon density and brightness of the X-ray laser. The gas bremsstrahlung is about $3 \times 10^{-5}$ times of that at SPring-8 and it is not so much sever. On the other hand, electron beam loss rate of the SACLA is about 10 times higher than that of SPring-8 top-up operation so that the bremsstrahlung due to electron beam loss is more

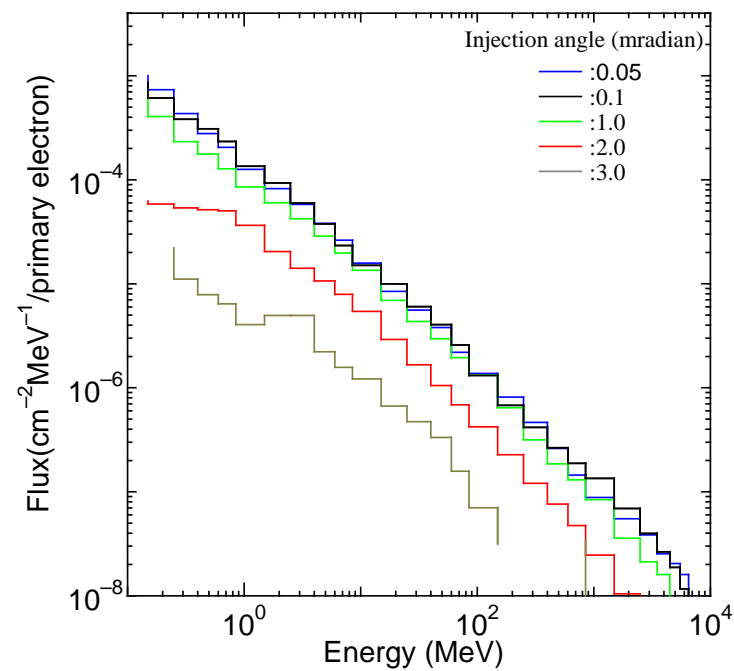

Figure 4. Bremsstrahlung spectra, depending on the injection angle to the accelerator components at the exit of the beamline hole of $90 \mathrm{~mm} \varphi$ to the optics hutch by using EGS4. Blue, black, green, red, and gray lines indicate the spectra for injection angels of $0.05,0.1,1.0,2.0$, and 3.0 mradian, respectively.
Table 1. Key parameters of SR and XFEL beamlines for radiation safety at SPring-8

\begin{tabular}{lll}
\hline Facility & $\begin{array}{l}\text { SPring-8 } \\
(\text { SR })\end{array}$ & $\begin{array}{l}\text { SACLA } \\
(\text { XFEL })\end{array}$ \\
\hline Current & $100 \mathrm{~mA}$ & $30 \mathrm{nA}$ \\
Energy & $8 \mathrm{GeV}$ & $8.5 \mathrm{GeV}(\mathrm{Max})$ \\
Undulator period & 140 (Typical) & $277 \times 18$ \\
Period length & $32 \mathrm{~mm}$ & $18 \mathrm{~mm}$ \\
Undulator & $0.78 \mathrm{Tesla}$ & $1.3 \mathrm{Tesla}$ \\
magnetic field & & \\
Straight section & $16.54 \mathrm{~m}$ (Typical) & $225 \mathrm{~m}$ \\
Gas pressure & $0.133 \mu \mathrm{Pa}$ & $1.0 \mu \mathrm{Pa}$ \\
Electron beam loss & $1.9 \times 10^{6} \mathrm{~s}^{-1}$ (normal) & $1.88 \times 10^{8} \mathrm{~s}^{-1}$ \\
rate (design value) & $(2.4 \mathrm{~mW})$ & $(255 \mathrm{~mW})$ \\
& $1.85 \times 10^{7} \mathrm{~s}^{-1}$ (top-up) & \\
& $(24 \mathrm{~mW})$ & $2 \mathrm{~cm}$ \\
\hline
\end{tabular}

important for the SACLA beamline. The bremsstrahlung spectrum due to electron beam loss at the exit of the beamline hole to the optics hutch as indicated in Figure 3 depends on the injection angle to the accelerator or undulator components, shown in Figure 4. Two collimators made of iron with the diameter of $13 \mathrm{~mm} \phi$ hole are installed in the front-end so that the aperture size of the SACLA beamline, defined 0.312 mradian. The bremsstrahlung that invades into the optics hutch has nearly an inverse energy spectrum, and it is almost saturated at the injection angle of 0.1 mradian with about $10^{-7}$ times the total power under a $0.1 \%$ beam loss. The shield walls, including local shields such as a local shield for a scatter and a gamma stop, were decided under the electron

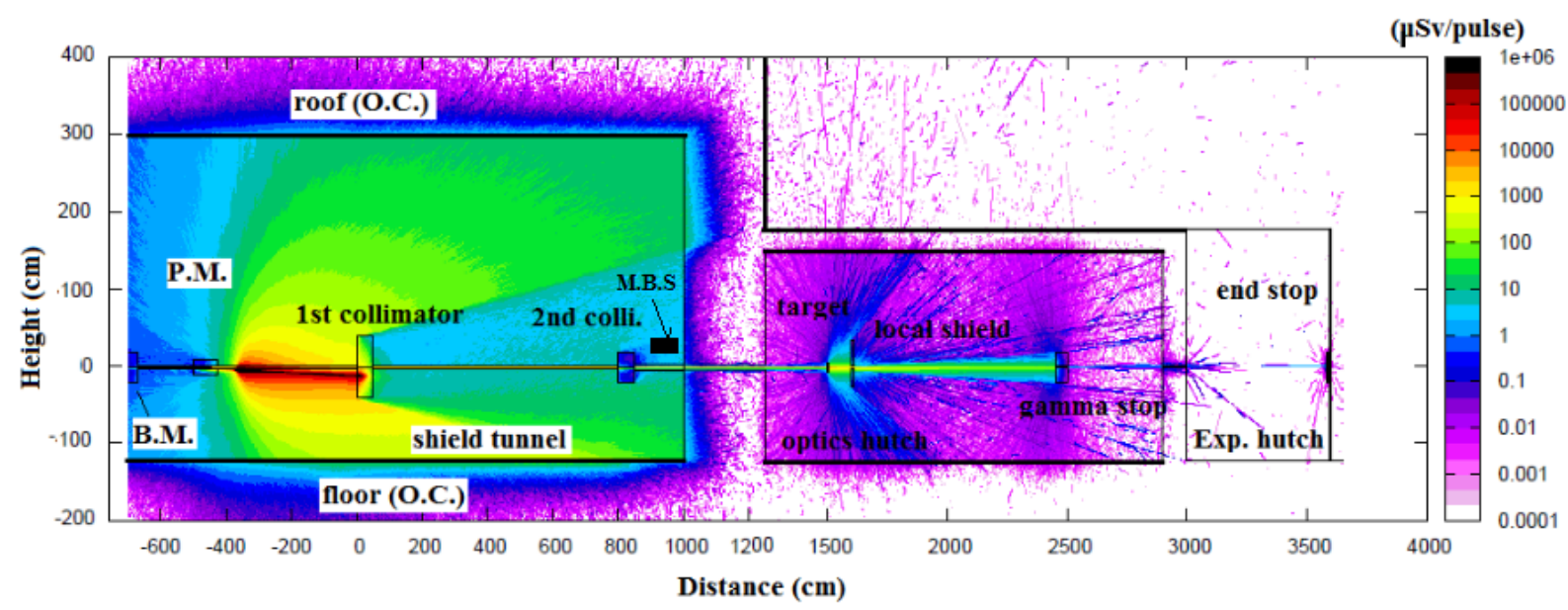

Figure 5. Leakage dose distributions during an accidental condition of the power loss of the dump bending magnet under operation of the experimental hutch. B.M and P.M. are the bending magnet of dump and the safety permanent magnet, respectively. M.B.S is the main beam shutter made of iron and tungsten with $40 \mathrm{~cm}$ thicknesses. 
beam conditions to be $80 \mathrm{~cm}$ of O.C. (ordinary concrete with density of more than $2.2 \mathrm{~g} / \mathrm{cm}^{3}$ ) for the side and roof and $100 \mathrm{~cm}$ of O.C. for the back wall.

A safety permanent magnet $(0.825 \mathrm{~T}, 73 \mathrm{~cm})$ was installed into the X-ray light beam axis for high- energy electrons never invading into the optics hutch. Figure 5 shows the dose distribution at the incident condition of the power loss of the dump bending magnet. In this case, one pulse $(0.5 \mathrm{nC})$ of $8.5 \mathrm{GeV}$ electrons hits the first collimator, and the bremsstrahlung produced by interactions between the electrons and a transport pipe invades into the optics hutch, and then hits a target, such as optical elements. For safety, $5 \mathrm{~cm}$ copper was assumed for the target. In the optics hutch, we installed a local shield and a gamma stop made of lead with thicknesses of $10 \mathrm{~cm}$ and $50 \mathrm{~cm}$, respectively. In this case, the off-set that the distance between the primary light beam axis and the second light beam axis to the experiment is set to $2 \mathrm{~cm}$. The perforated hole of the gamma stop is $1.2 \mathrm{~cm}$ so that a small amount of bremsstrahlung could go into experimental hutch during the DSS (downstream shutter), which opened as shown in the figure. The leakage dose dependence to the experimental hutch on the off-set is shown in Figure 6 at a loss of the dump bending magnet. It is clear that the leakage doses strongly depended on the off-set, which is also important for a leakage dose estimation, due to $0.1 \%$ electron beam loss. However, the off-set was determined by the performance of the optical elements and the space.

The shielding design of the experimental hutch walls was performed using STAC8 [8] for an X-ray laser and synchrotron radiation. FLUKA for high-energy radiation due to electron beam loss was also employed under the simulation conditions of the $2 \mathrm{~cm}$ off-set with a copper scatterer of $5 \mathrm{~cm}$ thickness conservatively. As results, we found that the iron with $1 \mathrm{~cm}$ thickness is sufficient for the side and the roof walls. Each back wall is required to be $3 \mathrm{~cm}$ of iron and the additional local shields made of lead with $0.5 \mathrm{~cm}$ thickness within a radius of $40 \mathrm{~cm}$, and $0.2 \mathrm{~cm}$ within a radius of $100 \mathrm{~cm}$. A movable end-stop made of graphite with $10 \mathrm{~cm}$ thickness and lead with $10 \mathrm{~cm}$ thickness was installed upstream of each back wall of the experimental hutch for direct beam shielding, and preventing heat damage due to the X-ray laser. The beam transport from the EH4 experimental hutch to the EH5 hutch, as shown in Figure 1 was performed by using a collimator made of tungsten and a beam transport pipe of which the diameter increased in stages towards the EH5 hutch.

\section{Safety system}

The radiation safety system at SACLA consists of a radiation monitoring system, an access control system and a beam containment system, including beam route controls and shields. The radiation monitors were set outside of the shield wall, including

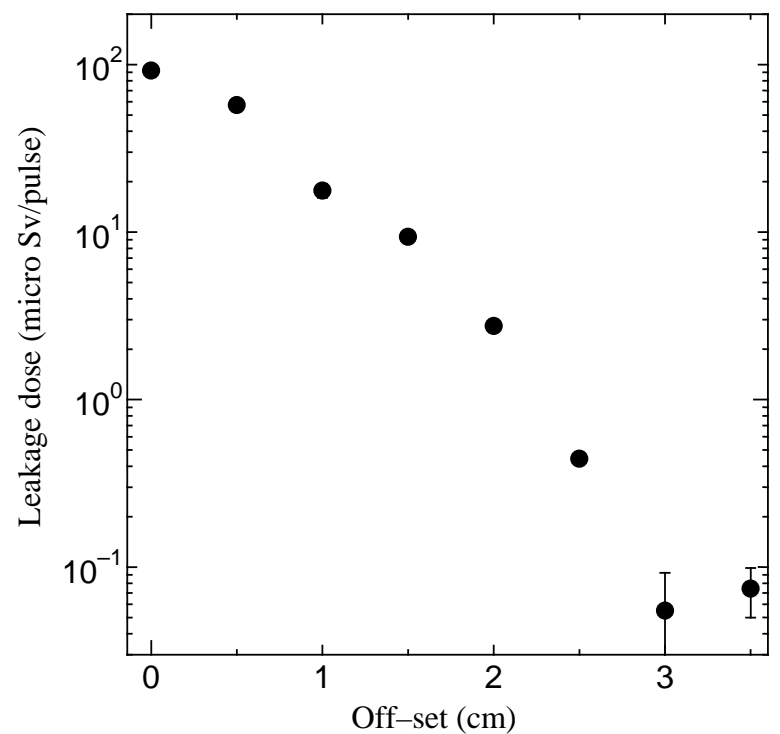

Figure 6. Leakage dose dependence on the off-set at the power loss of the SACLA dump bending magnet.

the boundary of the controlled area; personal keys and a door interlock system were employed for access control. These systems are linked organically by an interlock system and these are fundamentally the same as the SPring-8's system. In addition to the system, a beam halo monitor [9] and beam loss monitors [10] were installed within and around the electron transport pipes, respectively, to certify $0.1 \%$ or less beam loss rate at the undulator section.

\section{Conclusion}

The shielding design and safety systems for SACLA are described. SACLA was constructed to obtain the $\mathrm{X}$-ray laser with high quality and the shortest wavelength on the basis of the concepts of "compact" and "Japanese original technologies". The shielding design is also performed on the basis of the concept of compact, so that we make full use of a so called "local shield" for beamline shielding. For accelerator shielding, the design criterion of the site boundary is dominant. In addition to the general safety system, a halo monitor and beam-loss monitors were installed, which are effective to prevent increasing unwanted beam losses and to protect against a high leakage dose effectively. Since May 2012, the X-ray laser has been provided to experiments continuously for out-side users and in-house members without any radiation problems.

\section{References}

[1] T. Ishikawa, H. Aoyagi, T. Asaka, et al., A compact X-ray free-electron laser emitting in the sub-ångström region, Nat. Photon. 6 (2012), pp. 540-544.

[2] W.R. Nelson and T.M. Jenkins, The SHIELD 11 Computer code, SLAC-Report 737 (2005)

[3] W.R. Nelson, H. Hirayama and D.W.O.Rogers, 
The EGS4 code system, SLAC-265 (1985)

[4] A. Fasso, A. Ferrari, J. Ranft and P.R. Sala, FLUKA Proc.Monte Carlo 2000 Conf.

[5] Y. Asano, T. Itoga and X-M Marèchal, Radiation shielding aspects of the SCSS prototype XFEL facility, Nuclear Technology 168(2009), pp.387-390.

[6] Y. Asano, T. Bizen and X-M. Marèchal, Analyses of the factors for the demagnetization of permanent magnets caused by high-energy electron irradiation, J. Synchrotron Radiation 16 (2009), pp.317-324.

[7] Y. Asano, Characteristics of radiation safety for synchrotron radiation and X-ray free electron laser facilities, Radiation Protection Dosimetry DOI10.1093 (2011), pp.115-118.
[8] Y. Asano and N. Sasamoto, Development of shielding design code for synchrotron radiation beamline, Radiation Physics and Chemistry, Vol 44 No.1/2(1994)

[9] H. Aoyagi, Y. Asano, T. Itoga, N. Nariyama, T. Bizen, T. Tanaka and H. Kitamura, Pulse-mode measurement of electron beam halo using diamond-based detector, Physical Review SP Accelerators \& Beams 15 (2012), pp.022801.1-8.

[10]X.M. Marèchal, Y. Asano and T. Itoga, Design development, and operation of a fiber-base Cherenkov beam loss monitor at the SPring-8 Angstrom Compact Free Electron Laser, Nucl. Instr. Methods A 673 (2012) pp.32-45 